\title{
Produção científica qualificada: estudo de características acadêmicas de docentes dos Programas Stricto Sensu em Ciências Contábeis da região sul
}

Qualified scientific production: study of academic characteristics of teachers of the Stricto Sensu Programs in Accounting Sciences of the south region

\author{
Simone Rodrigues Zabolotny ${ }^{1}$ \\ ${ }^{1}$ Universidade Federal do Rio Grande | Rio Grande | RS | Brasil. Contato: \\ simonezabolotny@furg.br. ORCID: http://orcid.org/0000-0002-0965-5439
}

Anderson Betti Frare ${ }^{2}$

${ }^{2}$ Universidade Federal do Rio Grande | Rio Grande | RS | Brasil. Contato: anderson_betti_frare@hotmail.com. ORCID: http://orcid.org/0000-0002-4602-7394

Débora Gomes de Gomes ${ }^{3}$

${ }^{3}$ Universidade Federal do Rio Grande | Rio Grande | RS | Brasil. Contato: debora_furg@yahoo.com.br. ORCID: https://orcid.org/0000-0002-7955-0958

Rodrigo Nobre Fernandez ${ }^{4}$

${ }^{4}$ Universidade Federal de Pelotas | Pelotas | RS | Brasil. Contato: rodrigo.fernandez@ufpel.edu.br. ORCID: http://orcid.org/0000-0001-8596-2898

Resumo: Este estudo busca analisar se as características acadêmicas de docentes dos Programas de Pós-Graduação stricto sensu de Ciências Contábeis da região Sul influenciam na produção científica qualificada de artigos em periódicos. O estudo descritivo e quantitativo foi realizado através da coleta das informações nos Currículos Lattes, de cento e oito docentes, vinculados a oito Programas de Pós-Graduação stricto sensu em Ciências Contábeis da região Sul. Após a coleta das informações dos docentes (número de orientações, gênero, idade, formação) foi realizada a análise quantitativa, através da técnica de regressão com dados em painel. Nos resultados, verifica-se que não há como afirmar que a idade e o gênero influenciem na produção científica. Quanto as orientações, observa-se que a pontuação dos docentes acresce a cada orientação de mestrado, iniciação científica, graduação, especialização, em percentual de $7 \%, 5 \%, 3 \%, 2 \%$, respectivamente.

Palavras-chave: Produção científica. Docentes. Programa de Pós-Graduação.

Abstract: This study aims to analyze if the academic characteristics of professors of the Graduate Programs stricto sensu of Accounting Sciences in the Southern region influence the qualified scientific production of articles in journals. The descriptive and quantitative study was carried out through the collection of information in the Curriculum Lattes of one hundred and eight teachers, linked to eight Graduate Programs stricto sensu in Accounting in the Brazilian Southern region. After collecting information from teachers (number of orientations, gender, age, academic formation) quantitative analysis was performed using the regression technique with panel data. In the results, it is verified that there is no way to affirm that age and gender influence the scientific production. As for the orientations, it is observed that the teachers' score increases with each master's, scientific initiation, undergraduate, specialization orientation, in a percentage of $7 \%, 5 \%, 3 \%, 2 \%$, respectively.

Keywords: Scientific production. Teachers. Post-Graduated.

- Recebido em: 16 de janeiro de 2020 - Aprovado em: 7 de abril de 2020

DOI: http://dx.doi.org/ 10.1590/S1414-4077/S1414-40772020000200007

Este é um artigo publicado em acesso aberto sob uma licença Creative Commons

https://creativecommons.org/licenses/by-nc/4.0/ 


\section{Introdução}

Os Programas de Pós-Graduação stricto sensu, como parte integrante do ensino superior, buscam por meio da constante produção científica, o aperfeiçoamento e desenvolvimento da ciência. Assim, as produções de pesquisas acadêmicas e as suas publicações desempenham papel essencial para a construção e disseminação de novos conhecimentos (SILVA et al., 2012; SOARES; RICHARTZ; MURCIA, 2013; WAICZYK; ENSSLIN, 2013).

A Pós-Graduação stricto sensu, segundo Saviani (2000), possui um objetivo próprio, distinto daquele dos cursos que estão voltados para a formação profissional, pois tem como objetivo formar novos pesquisadores. Com relação à pesquisa em Contabilidade no Brasil, esta tem apresentado indicativos de crescimento, e esse fato deve-se principalmente ao atual momento da pesquisa, estimulada pelos cursos de Pós-Graduação em Contabilidade (SOARES et al., 2018; VIEIRA; ENSSLIN; SILVA, 2011).

Nesse sentido, para a manutenção dos níveis de qualidade dos programas de PósGraduação stricto sensu, conforme Silva et al. (2012), a Coordenação de Aperfeiçoamento de Pessoal de Nível Superior (CAPES) avalia periodicamente os programas, sendo a produtividade científica o indicador que possibilita a essa instituição avaliar o desempenho das instituições por meio de sua produção intelectual.

Dentro deste escopo, cabe destacar, a atuação dos docentes, principais atuantes na produção dos artigos científicos. Há uma conformidade que a produção acadêmica necessita das competências do pesquisador, e que para atingir um nível de excelência a produção precisa ser realizada constantemente (YAMAMOTO et al., 2012; MUELLER, 2000).

A produção científica, pode ser vista como uma das ferramentas para o desenvolvimento da sociedade (GARVEY, 1979; SOUZA; SILVA; ARAÚJO, 2012). Os artigos em periódicos representam um importante meio de divulgação da pesquisa acadêmica, uma vez que os seus resultados podem amplamente difundidos para a sociedade, devido a facilidade de divulgação e circulação (SOUZA; SILVA; ARAÚJO, 2012).

Diante do exposto, constata-se que a pesquisa científica desempenha importante papel para a elevação do conhecimento, pois contribui para a construção e a evolução de determinada área de conhecimento, além de contribuir para a sociedade, por meio do desenvolvimento de novos estudos (SOARES; RICHARTZ; MURCIA, 2013). 
Adentre este contexto, entende-se que o docente constitui parte do processo de construção da pesquisa nos Programas de Pós-Graduação stricto sensu. Sendo assim, conhecer as suas características e mapear as suas produções científicas contribuiria segundo Imasato, Perlin e Borenstein, (2017), para elucidar se existe relação entre as características do docente e a sua produção científica, e conhecer essa relação contribuiria para adicionar novos aspectos às discussões existentes na literatura e qualificá-las.

Embora existam estudos pregressos que se propõem a investigar a produção científica em contabilidade (LEITE FILHO, 2010; PELEIAS et al., 2010; SILVA et al., 2012; SOARES; RICHARTZ; MURCIA, 2013), poucos trabalhos relacionaram as características dos docentes e a produção científica (BARBOSA; BARROS, 2010; IMASATO; PERLIN; BORENSTEIN, 2017; MARTINS; LUCENA, 2014; RORSTAD; AKSNES, 2015; SANTANA; NGANGA; LEAL, 2014).

Estudos pregressos indicam lacunas de pesquisas existentes no campo empírico, tais como o de Barbosa e Barros (2010), que propõem novas pesquisas relacionando o perfil dos autores com a produção científica publicados em periódicos. Assim surge o seguinte problema de pesquisa: Qual a influência das características acadêmicas dos docente stricto sensu em sua produção científica qualificada? Em estudo realizado por Yamamoto et al. (2012), os autores informaram que a produção científica se mostrou como um relevante indicador para a PósGraduação, fato que fortalece o problema de pesquisa proposto.

De forma a alcançar a resposta da questão de pesquisa proposta, este estudo tem como objetivo analisar se as características acadêmicas (número de orientações, gênero, idade, curso de formação) de docentes dos Programas de Pós-Graduação stricto sensu de Ciências Contábeis da região Sul influenciam na produção científica qualificada de artigos em periódicos com Qualis (A1, A2, B1, B2, B3, B4 e B5). Para tanto, os dados serão analisados mediante regressão com dados em painel.

Inerentemente ao escopo do objetivo central, instiga-se especificamente a: (i) identificar os docentes que fazem parte dos Programas de Pós-Graduação stricto sensu em Contabilidade no Rio Grande do Sul, Santa Catarina e Paraná; (ii) coletar as informações nos currículos dos docentes que compõem a amostra, no período de 2014 a 2018 e (iii) analisar as características acadêmicas coletadas.

A pesquisa justifica-se, devido à relevância da produção científica para a evolução dos Programas de Pós-Graduação stricto sensu em Contabilidade e o envolvimento do docente para concretização da produção científica (COTTINGHAM; HUSSEY, 2000; LEITE FILHO, 2010; OLIVEIRA, 2002). Segundo Soares et al. (2018) nas avaliações realizadas pela CAPES, a 
produção científica tem sido um peso relevante. Assim, faz-se necessário compreender os elementos desse processo avaliativo.

O estudo promove implicações teóricas e práticas. Sob o viés prático, as contribuições permeiam achados pertinentes para os Programas de Pós-Graduação stricto sensu em Contabilidade e para os próprios docentes, podendo subsidiar informações relevantes para o planejamento de ambos. Ademais, a ótica de contribuições teórica concerne a discussão de como as orientações refletem na produção acadêmica qualificada dos docentes.

Este artigo está estruturado em cinco seções, sendo esta primeira, que abrange a contextualização do tema, apresenta os achados pregressos de estudos anteriores, destaca a lacuna de pesquisa, apresenta o problema e objetivo da pesquisa, seguido da justificativa e contribuição do estudo. A seção dois abrange o aporte teórico do estudo. A seção três apresenta o delineamento metodológico. A seção quatro descreve os resultados encontrados, bem como a comparação destes com a literatura e os estudos anteriormente realizados. Por fim, a quinta seção apresenta as considerações finais do estudo, seguida pelas referências utilizadas.

\section{Fundamentação teórica}

Concatenado a construção da fundamentação teórica, a presente seção segrega-se em três momentos. Inicialmente são discutidos aspectos relativos aos programas de pós-graduação e produção científica. Na sequência, são apresentados os parâmetros de avaliação da produção científica, adentre o âmbito e cenários fornecidos pela CAPES. Por último, promove-se a discussão acerca de estudos pregressos que entoam na temática do artigo.

\subsection{Programas de Pós-Graduação e Produção Científica}

A Pós-Graduação no Brasil iniciou-se a partir do Parecer 977/65, de autoria de Newton Sucupira, que teve como marco inicial a conceituação da Pós-Graduação. Posteriormente, em 1969, o mesmo conselheiro foi o relator do parecer 77/69 que regulamentou e implantou a PósGraduação. Foi em 1965 que surgiu o primeiro curso de Pós-Graduação no Brasil, na Pontifícia Universidade Católica do Rio de Janeiro, no nível de mestrado (SAVIANI, 2000).

No que tange ao primeiro Programa de Pós-Graduação em Contabilidade iniciou-se em 1970, com a criação do curso de mestrado da Faculdade de Economia, Administração e Contabilidade, na Universidade de São Paulo (FEA/USP). No mesmo ano, foi criado o Programa de Pós-Graduação em Ciências Contábeis da Fundação Getúlio Vargas, no Rio de 
Janeiro, e foi somente em 1978, que o primeiro curso de doutorado foi implantado na FEA/USP. (CAPES, 2018).

Durante a década de 1980 nenhum novo Programa de Pós-Graduação stricto sensu foi implantado. Peleias et al. (2007) apontam alguns motivos para o surgimento de novos programas a partir da década de 1990: (i) as exigências da Lei n. 9.394/96, para que pelo menos um terço do corpo docente das instituições de ensino superior, fossem de professores com titulação mínima de Mestrado, (ii) a exigência da presença de professores em tempo integral dedicados à docência e à pesquisa, (iii) a ampliação de cursos de Ciências Contábeis, (iv) o maior número de professores com titulação de doutorado em Ciências Contábeis, (v) a atuação de professores doutores em outras áreas que não em Ciências Contábeis nos programas stricto sensu em Contabilidade, o que em parte reduziu a restrição de haver apenas um Doutorado na área no País.

No entanto, foi a partir do século XXI que o número de Programas de Pós-Graduação scricto sensu em Contabilidade aumentou expressivamente. De acordo com informações da CAPES, em 2009, existiam 21 cursos de mestrado e 4 cursos de doutorado (CAPES, 2009), já em 2016 o número de cursos aumentou para 27 de mestrado e 12 de doutorado. Nota-se que o número de doutorados aumentou expressivamente, em especial, motivado pela consolidação de cursos de mestrado que iniciaram por volta dos anos 2000 (CAPES, 2016).

Assim, em decorrência da expansão dos Programas de Pós-Graduação em Ciências Contábeis, espera-se um aumento da produção científica, já que o foco dos programas é a formação de pesquisadores e docentes (SILVA et al., 2012).

Leite Filho (2010) acrescenta que se tem observado um crescimento da pesquisa e da publicação científica, devido aos seguintes motivos: aumento de professores e pesquisadores titulados, aumento na participação dos docentes em congressos nacionais e internacionais, expansão dos cursos de Pós-Graduação (lato e stricto sensu) e da pressão exercida pela CAPES, já que um dos critérios de avaliação para as universidades inclui a produção intelectual dos docentes e pesquisadores.

Segundo informações da CAPES, a produção científica, na base Scopus, na temática Business, Management and Accounting passou da $30^{\mathrm{a}}$ posição em 1998 , para a $12^{\mathrm{a}}$ posição em 2015, esse aumento na publicação demonstrou que se tem dado mais valor para as publicações em periódicos do que as publicações em eventos científicos e congressos (CAPES, 2016).

Além disso, o aumento da produção científica possibilitou uma maior presença de trabalhos de pesquisa em Contabilidade em importantes eventos brasileiros como também 
internacionais, além da criação de eventos específicos, como o Congresso USP de Controladoria e Contabilidade (PELEIAS et al., 2007).

Apesar dos diversos veículos de divulgação da produção científica, segundo Imasato, Perlin e Borenstein (2017), os artigos publicados em periódicos indexados têm recebido mais atenção nas últimas décadas, devido aos seguintes motivos: o registro do número de citações, o que pode indicar a recepção e o impacto do trabalho publicado; o cálculo do fator de impacto da revista, realizado com base na extração da média de citações dos artigos publicados; estratificação das publicações, demonstrando a percepção de qualidade ou o prestígio do periódico.

A CAPES concerne como órgão responsável por avaliar os Programas de PósGraduação, assim como credenciar os novos cursos. Conhecer os critérios de avaliação desse órgão torna-se importante, pois a partir desse conhecimento, se torna possível compreender como os programas estão qualificados e traçar objetivos de como melhorar (SOARES et al., 2018). Nesta perspectiva, a próxima subseção apresenta quais são os critérios utilizados para avaliação desses programas, mediante parâmetros propostos pela CAPES.

\subsection{Avaliação dos Programas de Pós-Graduação}

A CAPES realiza quadrienalmente a avaliação dos Programas de Pós-Graduação no Brasil. Os critérios para a realização da avaliação estão dispostos em documentos orientadores de cada área de avaliação. Os cursos de Ciências Contábeis estão incluídos na área de Administração Pública e de Empresas, Ciências Contábeis e Turismo (CAPES, 2016). Segundo a ficha de avaliação para o quadriênio 2013-2016, os quesitos analisados estão dispostos conforme o Quadro 1.

Quadro 1 - Ficha de avaliação para o quadriênio 2013-2016

\begin{tabular}{|l|c|}
\hline \multicolumn{1}{|c|}{ Quesitos } & Participação na nota total \\
\hline 1. Proposta do programa & $50 \%$ \\
\hline 2. Corpo docente & $20 \%$ \\
\hline 3. Corpo discente, teses e dissertações & $35 \%$ \\
\hline 4. Produção intelectual & $35 \%$ \\
\hline 5. Inserção social & $10 \%$ \\
\hline
\end{tabular}

Fonte: Adaptado de CAPES (2016).

Constata-se, por meio da análise do Quadro 1, que a produção científica configura-se como um fator relevante na avaliação de um programa de Pós-Graduação em Contabilidade no Brasil. Destaca-se que para fins de avaliação, a CAPES apenas contabiliza os artigos que foram 
publicados em periódicos, excluindo-se para fins de contagem as produções em eventos científicos e em congressos (SOARES; RICHARTZ; MURCIA, 2013).

Como critérios para a mensuração das produções científicas em periódicos, a CAPES utiliza o Qualis para classificar os periódicos. O Qualis configura-se como um ranqueamento dos periódicos de acordo com a avaliação do comitê de cada área, de forma a estratificar em ordem decrescente os indicativos de qualidades, sendo: A1 o mais elevado, seguido de A2; B1; B2; B3; B4; B5; e, por último, C - com pontuação zero (SILVA et al., 2012).

O Quadro 2 apresenta os estratos nos quais os periódicos podem ser listados no Qualis e a pontuação respectiva de cada nível para a área de Administração, Ciências Contábeis e Turismo.

\section{Quadro 2 - Qualis Capes}

\begin{tabular}{|c|c|c|c|c|c|c|c|c|}
\hline Estrato & A1 & A2 & B1 & B2 & B3 & B4 & B5 & C \\
\hline Pontuação & 100 & 80 & 60 & 50 & 30 & 20 & 10 & 0 \\
\hline
\end{tabular}

Fonte: Adaptado de CAPES (2017).

Segundo Murcia, Rosa e Borba (2013) os primeiros estratos como A1, A2 e B1 são considerados de alto impacto na área de Administração, Ciências Contábeis e Turismo. Em sua maioria os periódicos A1 e A2 são internacionais e periódicos B1 são cotados como os melhores nacionais.

Conforme enfatizado pela CAPES (2017), o aperfeiçoamento do conhecimento e a produção científica configuram os seus objetivos. Assim, a pesquisa científica torna-se produto de todo o esforço dos trabalhos dos Programas de Pós-Graduação para a elevação do conhecimento. E, para manter a qualidade do ensino stricto sensu e fiscalizar o desempenho dos Programas a CAPES foi criada.

Para enfatizar essa necessidade, Mesquita et al. (2013) informam que a avaliação dos Programas de Pós-Graduação iniciou-se em 1976, pela CAPES, com o objetivo de desenvolvimento da Pós-Graduação, bem como para contribuir para a pesquisa científica brasileira. Torna-se importante então que os Programas de Pós-Graduação mantenham um padrão de qualidade e uma produção constante para atender os preceitos da CAPES para manterem a sua avaliação positiva junto a mesma.

\subsection{Estudos pregressos}

A pesquisa realizada por Barbosa e Barros (2010) visou analisar bibliometricamente o perfil dos autores em contabilidade a partir dos artigos publicados no Congresso da 
Universidade de São Paulo (USP) de Controladoria e Contabilidade e no Congresso da Associação Nacional dos Programas de Pós-Graduação em Ciências Contábeis (ANPCONT), no período de 2009 e 2010. Mediante análise do número de autores por artigo, examinaram os autores e as instituições mais prolíficas, o gênero e o nível de formação dos autores.

Adicionalmente o estudo de Santana, Nganga e Leal (2014) pesquisou o perfil e mapeou a produção científica dos docentes dos cursos de Pós-Graduação stricto sensu em Ciências Contábeis no Brasil que obtiveram nota superior a quatro na avaliação trienal da CAPES. As seguintes características foram analisadas: qualificação, o ano de início de atuação do docente na IES; as linhas e projetos de pesquisa; as publicações em congressos, periódicos, livros, capítulos de livros e orientações.

Na área de Administração o estudo de Imasato, Perlin e Borenstein (2017) propôs analisar as características dos docentes que atuam na área de Administração no Brasil e as suas respectivas produções científicas, de modo a identificar fatores que expliquem esse aspecto da produtividade científica.

No contexto internacional, Rorstad e Aksnes (2015), analisaram se o desempenho científico, em termos de pontuação científica foi influenciado pelo gênero, idade e posição acadêmica dos pesquisadores, o estudo envolveu quase 12.400 pesquisadores universitários noruegueses e mostrou que a posição acadêmica é mais importante do que a idade e sexo.

Martins e Lucena (2014) buscaram identificar o perfil e as práticas dos docentes dos programas de pós-graduação em Contabilidade (PPGC) no que se refere à produção científica dos mesmos, sendo a análise realizada a partir da aplicação de um questionário, no qual o docente foi questionado a respeito da pontuação alcançada no triênio, divisão de autoria, escolha dos periódicos para publicação, bem como principal meio de alcance da pontuação científica.

\section{Delineamento metodológico}

Como esse estudo tem por objetivo analisar se as características acadêmicas de docentes dos Programas de Pós-Graduação stricto sensu de Ciências Contábeis da região Sul influenciam na produção científica qualificada de artigos em periódicos, sua classificação, quanto aos objetivos, enquadra-se como descritiva, segundo os pressupostos de Gil (2002). Quanto aos procedimentos, a pesquisa enquadra-se como documental, ver Martins e Theóphilo (2018) e quanto à abordagem do problema, a pesquisa se enquadra em quantitativa, conforme o descrito em Martins e Theóphilo (2018). 
A população deste estudo compõe-se por 108 docentes dos Programas de PósGraduação stricto sensu em Ciências Contábeis da região Sul do Brasil, compreendendo os seguintes Institutos de Ensino Superior: do estado do Rio Grande do Sul: da Universidade do Vale dos Sinos (UNISINOS), da Universidade Federal do Rio Grande do Sul (UFRGS) e Universidade Federal do Rio Grande (FURG); do estado de Santa Catarina: Universidade Comunitária da Região de Chapecó (UNOCHAPECÓ), da Universidade Federal de Santa Catarina (UFSC) e da Universidade Regional de Blumenau (FURB) e do estado do Paraná: da Universidade Estadual do Oeste do Paraná (UNIOESTE) e da Universidade Federal do Paraná (UFPR).

A relação de docentes foi obtida no site dos Programas de Pós-Graduação stricto sensu, em 01 de maio de 2019. Foram objetos de análise todos os docentes apontados pela relação dos programas, ou seja, aqueles que possuem vínculo permanente, ou colaborador e/ou visitante.

\subsection{Coleta de dados}

A coleta de dados foi realizada por meio da Plataforma Lattes e da Plataforma Sucupira, compreendendo o período de 2014 a 2018. Para a coleta das informações (gênero; formação acadêmica/titulação; e orientações concluídas), utilizou-se a Plataforma Lattes; para a coleta das idades dos docentes, utilizou-se a Plataforma Sucupira; para a coleta dos artigos completos publicados em periódicos, utilizou-se a Plataforma Lattes.

No que tange à coleta dos dados das produções científicas, conforme descrito na última assertiva do parágrafo anterior, esta foi operacionalizada mediante o download dos currículos dos docentes na Plataforma Lattes, em formato $x m l$, para então serem importados no package GetLattesData (PERLIN, 2019), nos softwares R 3.6.0 e RStudio. Com o auxílio deste package, tornou-se possível de forma ágil, a tabulação dos dados em planilha eletrônica, de maneira a organizar as informações dispostas nos currículos em questão.

\subsection{Estratégia empírica}

Para análise da pontuação dos periódicos utilizou-se a estratificação do Qualis juntamente com a pontuação estabelecida pela CAPES (2016). Os conceitos dos periódicos foram obtidos pelo estrado Qualis/CAPES da área de Administração, Contabilidade e Turismo, por meio do acesso ao documento: Considerações sobre Qualis Periódicos, disponível no site da CAPES. São objetos de análise apenas as publicações em periódicos realizadas no período de 2014 a 2018, registrados no Currículo Lattes dos docentes. 
Devido a característica de contagem da variável, pontuação de cada docente no ano t, para a realização da análise quantitativa utilizou-se do modelo de regressão de Poisson. De acordo com Cameron e Trivedi (2009) esse modelo de regressão de especifica a contagem $\mathcal{Y}$ para ter uma média condicional da forma exponencial.

$$
\mathrm{E}(\mathcal{Y} \mid \mathrm{X})=\exp \left(\mathrm{X}^{\prime} \beta\right)
$$

Isso garante que a média condicional seja positiva, o que deve ser o caso de qualquer variável aleatória que esteja restrita a não ser negativa.

O ponto de partida para a análise de contagem é a distribuição de Poisson, com a função de massa de probabilidade $f(\mathcal{Y} \mid \mathrm{X})=e^{-\mu} \mu^{y} / \mathcal{Y}$ ! Substituindo em $\mu_{i}==\exp \left(x_{i}^{\prime} \beta\right)$ de (1) dá a densidade condicional para i-ésima observação. Isso, por sua vez, dá a função de logverossimilhança $Q(\beta) \sum_{i=1}^{N}\left\{-\exp \left(x_{i}^{\prime} \beta\right)+\mathcal{Y}_{i} x_{i}^{\prime} \beta-\ln \mathcal{Y}_{i} !\right\}$, que é maximizada pelo estimador de máxima verossimilhança (MLE).

Ao realizar-se a estimação de uma variável de contagem $\mathcal{Y}_{i t}$ para o indivíduo $i$ no período $t$, isto é, na estrutura de dados em painel torna-se necessário utilizar o estimador de Poisson que se adeque a essa forma. Assim, o modelo de efeitos fixos individuais de Poisson, considera que a heterogeneidade individual medida por $\alpha_{i}$ é possivelmente correlacionada com $x_{i t}$.

Desta forma, em painéis curtos (em que o número de indivíduos é estritamente maior que o período amostral a ser utilizado) é necessário eliminar $\alpha_{i}$ antes de estimar $\beta$. Esse parâmetro pode ser eliminado ao utilizar-se o estimador de log-verossimilhança condicional. As condições de primeira ordem deste estimador são as seguintes:

$$
\sum_{i=1}^{N} \sum_{t=1}^{T} x_{i t}\left(\mathcal{Y}_{i t}-\frac{\lambda_{i t}}{\lambda_{i}} y_{i}\right)=0(2)
$$

Em que: $\lambda_{i t}=\exp \left(x_{i t}^{\prime} \beta\right)$ e $\lambda_{i}=T^{-1} \sum_{t} \exp \left(x_{i t}^{\prime} \beta\right)$.

O estimador de efeitos fixos de Poisson é, portanto, consistente se $\mathrm{E}\left(\mathcal{Y}_{i t} \mid \alpha_{i}\right.$, $\left.x_{i}, \ldots, x_{i T}\right)=\alpha_{i} \exp \left(x_{i t}^{\prime} \beta\right)$ porque então o lado esquerdo de (2) tem o valor esperado de zero.

\section{Análise dos resultados}

Para a seção em questão, as análises são segregadas em: (i) análise descritiva; e (ii) análise quantitativa, a qual abarca as regressões dos dados em painel. 


\subsection{Análise descritiva}

Esta seção faz a análise dos dados obtidos por meio da apreciação das informações dos Currículos Lattes dos docentes dos Programas de Pós-Graduação stricto sensu pertencentes à amostra deste estudo, no período de 2014 a 2018. A Tabela 1, apresenta o quantitativo de docentes por Programa de Pós-Graduação stricto sensu e a distribuição desses profissionais por gênero.

Tabela 1 - Distribuição dos docentes quanto ao gênero

\begin{tabular}{cccccccc}
\hline Programa Stricto Sensu & Docentes (N) & $\boldsymbol{\%}$ & Nível & Masculino & \% & Feminino & \% \\
\hline UNISINOS & 14 & 12,73 & M/D & 11 & 78,57 & 3 & 21,43 \\
UFRGS & 15 & 13,64 & M & 9 & 60,00 & 6 & 40,00 \\
FURG & 9 & 8,18 & M & 4 & 44,44 & 5 & 55,56 \\
UNIOESTE & 14 & 12,73 & M & 8 & 57,14 & 6 & 42,86 \\
UFPR & 12 & 10,91 & M/D & 9 & 75,00 & 3 & 25,00 \\
UNOCHAPECÓ & 10 & 9,09 & M & 8 & 80,00 & 2 & 20,00 \\
UFSC & 19 & 17,27 & M/D & 13 & 68,42 & 6 & 31,58 \\
FURB & $(17) 15$ & 15,45 & M/D & 10 & 66,67 & 5 & 33,33 \\
\hline Total & $\mathbf{1 0 8}$ & $\mathbf{1 0 0 \%}$ & - & $\mathbf{7 2}$ & $\mathbf{6 6 , 6 7 \%}$ & $\mathbf{3 6}$ & $\mathbf{3 3 , 3 3 \%}$ \\
\hline
\end{tabular}

Fonte: Dados da pesquisa.

Por meio dos dados apresentados na Tabela 1, observa-se que os docentes do estudo são vinculados a oito Programas de Pós-Graduação stricto sensu, sendo que quatro destes programas possuem somente o nível mestrado (UFRGS; FURG; UNIOESTE e UNOCHAPECÓ) e os outros quatro programas possuem os níveis mestrado e doutorado (UNISINOS; UFPR; UFSC e FURB).

Cabe mencionar que, dois docentes da amostra são vinculados ao programa da UFSC e da FURB, assim a amostra do estudo é de 108 docentes e não de 110 docentes. Quanto ao gênero, observa-se a predominância do gênero masculino, uma vez que, dos 108 docentes analisados, 66,67\% são do sexo masculino e 33,33\% do sexo feminino. A UNOCHAPECÓ se destaca, pois, possui do total dos docentes do seu quadro, $80 \%$ do sexo masculino. A UNISINOS e a UFSC também se destacam, pois possuem 78,57\% e 68,42\% respectivamente. $\mathrm{Na}$ pesquisa de Santana, Nganga e Leal (2014) os resultados apontaram também a predominância de docentes do sexo masculino.

Por meio da Tabela 2, deslumbra-se a área de qualificação dos docentes vinculados aos programas de pós-graduação, quanto ao doutorado. 
Tabela 2 - Área de Qualificação dos Docentes

\begin{tabular}{cccccc}
\hline Programa Stricto Sensu & Área de Negócios & $\mathbf{\%}$ & Outros & \% & Total \\
\hline UNISINOS & 10 & 71,43 & 4 & 28,57 & 14 \\
UFRGS & 14 & 93,33 & 1 & 6,67 & 15 \\
FURG & 7 & 77,78 & 2 & 22,22 & 9 \\
UNIOESTE & 10 & 71,43 & 4 & 28,57 & 14 \\
UFPR & 8 & 66,67 & 4 & 33,33 & 12 \\
UNOCHAPECÓ & 6 & 60,00 & 4 & 40,00 & 10 \\
UFSC & 8 & 42,11 & 11 & 57,89 & 19 \\
FURB & 9 & 60,00 & 6 & 40,00 & 15 \\
\hline Total & $\mathbf{7 2}$ & $\mathbf{6 6 , 6 7 \%}$ & $\mathbf{3 6}$ & $\mathbf{3 3 , 3 3 \%}$ & $\mathbf{1 0 8}$ \\
\hline
\end{tabular}

Fonte: Dados da pesquisa.

Observa-se, pela visualização da Tabela 2, que existe o predomínio de formação na área de negócios, ou seja, Contabilidade, Administração, Economia, Finanças e Agronegócios, totalizando um percentual de $66,67 \%$ e os outros docentes da amostra possuem qualificação em outras áreas, como nas áreas de Educação, Engenharias e Psicologia, totalizando um percentual de 33,33\%. Percebe-se, que do total de docentes vinculados aos Programas de Pós-Graduação stricto sensu a UFRGS possui 93,33\% dos docentes com formação nas áreas de Contabilidade, Administração, Economia, Finanças e Agronegócios, já a UFSC possui dos dezenove docentes do seu quadro, onze com formação em outras áreas, como Doutorado em Engenharia de Produção, Engenharia e Gestão do Conhecimento, Engenharia Ambiental, correspondendo um total de $57,89 \%$.

A predominância de docentes com doutorado nas áreas de negócios corrobora achados nos estudos de Santana, Nganga e Leal (2014) e Leite Filho (2010), que verificaram que a maioria dos docentes possuíam qualificação nas áreas de Contabilidade, Economia e Administração. Com auxílio da Tabela 3, evidencia-se a pontuação das publicações dos docentes vinculados aos Programas de Pós-Graduação stricto sensu em estudo, no período de 2014 a 2018.

Tabela 3 - Pontuação dos docentes por Programa de Pós-Graduação stricto sensu

\begin{tabular}{ccccccccccc}
\hline $\begin{array}{c}\text { Programa Stricto } \\
\text { Sensu }\end{array}$ & $\mathbf{2 0 1 4}$ & $\mathbf{\%}$ & $\mathbf{2 0 1 5}$ & $\boldsymbol{\%}$ & $\mathbf{2 0 1 6}$ & $\boldsymbol{\%}$ & $\mathbf{2 0 1 7}$ & $\mathbf{\%}$ & $\mathbf{2 0 1 8}$ & $\boldsymbol{\%}$ \\
\hline UNISINOS & 2360 & 8,54 & 3200 & 12,89 & 1510 & 6,10 & 2740 & 11,41 & 2240 & 9,67 \\
UFRGS & 1320 & 4,78 & 1260 & 5,07 & 1560 & 6,30 & 2070 & 8,62 & 1490 & 6,43 \\
FURG & 530 & 1,92 & 1010 & 4,07 & 1060 & 4,28 & 930 & 3,87 & 720 & 3,11 \\
UNIOESTE & 2280 & 8,25 & 2000 & 8,05 & 2910 & 11,75 & 2270 & 9,45 & 2460 & 10,62 \\
UFPR & 1710 & 6,19 & 1340 & 5,40 & 1640 & 6,62 & 960 & 4,00 & 1490 & 6,43 \\
UNOCHAPECÓ & 2300 & 8,33 & 2400 & 9,67 & 1780 & 7,19 & 2670 & 11,12 & 3110 & 13,43
\end{tabular}




\begin{tabular}{ccccccccccc} 
UFSC & 9390 & 34,00 & 7070 & 28,47 & 7750 & 31,30 & 5930 & 24,70 & 6470 & 27,94 \\
FURB & 7730 & 27,99 & 6550 & 26,38 & 6550 & 26,45 & 6440 & 26,82 & 5180 & 22,37 \\
\hline Total & $\mathbf{2 7 6 2 0}$ & $\mathbf{1 0 0 \%}$ & $\mathbf{2 4 8 3 0}$ & $\mathbf{1 0 0 \%}$ & $\mathbf{2 4 7 6 0}$ & $\mathbf{1 0 0 \%}$ & $\mathbf{2 4 0 1 0}$ & $\mathbf{1 0 0 \%}$ & $\mathbf{2 3 1 6 0}$ & $\mathbf{1 0 0 \%}$ \\
\hline
\end{tabular}

Fonte: Dados da pesquisa

Constatou-se, a partir da análise da Tabela 3 que as produções em periódicos dos docentes do Programas em Pós-Graduação stricto sensu não seguiram uma distribuição uniforme. Os docentes dos Programas da UFSC e da FURB apresentaram-se mais produtivos ao longo dos cinco anos da análise, comparados aos outros docentes dos seis Programas de PósGraduação stricto sensu, representando mais de 55,52\% da produção e os outros Programas representaram 44,48\%. Tais resultados são convergentes com a pesquisa de Santana, Nganga e Leal (2014) que também encontraram a centralização da produção dos docentes vinculados aos Programas da UFSC e FURB.

A Tabela 4 evidencia o quantitativo de orientações dos docentes vinculados aos Programas de Pós-Graduação stricto sensu.

Tabela 4 - Distribuição das orientações concluídas pelos docentes (2014- 2018)

\begin{tabular}{ccccccc}
\hline $\begin{array}{c}\text { Programa } \\
\text { Stricto Sensu }\end{array}$ & $\begin{array}{c}\text { Iniciação } \\
\text { Científica }\end{array}$ & Graduação & Especialização & Mestrado & Doutorado & Pós Doc \\
\hline UNISINOS & 8 & 107 & 44 & 159 & 20 & 4 \\
UFRGS & 15 & 191 & 122 & 45 & 9 & 0 \\
FURG & 30 & 43 & 53 & 20 & 0 & 0 \\
UNIOESTE & 111 & 136 & 106 & 98 & 4 & 1 \\
UFPR & 32 & 71 & 51 & 70 & 17 & 1 \\
UNOCHAPECÓ & 44 & 179 & 154 & 50 & 0 & 0 \\
UFSC & 36 & 364 & 60 & 102 & 29 & 9 \\
FURB & 12 & 50 & 0 & 108 & 23 & 3 \\
\hline Total & $\mathbf{2 8 8}$ & $\mathbf{1 1 4 1}$ & $\mathbf{5 9 0}$ & $\mathbf{6 5 2}$ & $\mathbf{1 0 2}$ & $\mathbf{1 8}$ \\
\hline \% & $\mathbf{1 0 , 3 2}$ & $\mathbf{4 0 , 8 8}$ & $\mathbf{2 1 , 1 4}$ & $\mathbf{2 3 , 3 6}$ & $\mathbf{3 , 6 5}$ & $\mathbf{0 , 6 4}$ \\
\hline
\end{tabular}

Fonte: Dados da pesquisa.

Nota-se a partir da análise da Tabela 4, que os docentes analisados orientam mais alunos em trabalhos de conclusão de curso, na graduação, representando um percentual de 44,8\%. Esse fato, indica que os docentes atuam nos Programas de Pós-Graduação stricto sensu, e também continuam atuando com orientações na graduação. Quanto às orientações de Mestrado, destacam-se as orientações dos docentes dos Programas UNISINOS, com 159 orientações, seguidas das orientações da FURB, com 108 orientações, e da UFSC com 102 orientações.

Observa-se o número reduzido de orientações do nível de Doutorado, representando o total de 3,65\%. Cabe mencionar, que dos oito Programas de Pós-Graduação stricto sensu, apenas quatro possuem os dois níveis, Mestrado e Doutorado. O primeiro Programa com nível 
de Doutorado no Sul foi da FURB, desde 01/01/2008, seguido da UNISINOS, desde 01/01/2013, UFSC, desde 01/01/2013, e por último da UFPR, desde 01/01/2014. A presença de orientações de Doutorado ou Pós-Doc, em Programas que não possuem o nível de Doutorado, podem indicar que os docentes destes Programas realizam orientações em outros Programas.

\subsection{Análise quantitativa}

A seguir apresentam-se os resultados das regressões dos determinantes das Pontuações dos docentes dos Programas de Pós-Graduação stricto sensu em Ciências Contábeis, do Sul do Brasil. Destaca-se que se tem um painel balanceado com 108 docentes para o período de 2014 a 2018. O Quadro 3 evidencia as variáveis utilizadas no estudo, bem como a sua descrição.

Quadro 3 -Descrição das variáveis analisadas

\begin{tabular}{|c|c|}
\hline Variável & Descrição da Variável \\
\hline Idade & Idade do Docente \\
\hline Gênero & Gênero do Docente \\
\hline Mestrado & Orientações de Mestrado \\
\hline Doutorado & Orientações de Doutorado \\
\hline Pós-Doutorado & Orientações de Pós-Doutorado \\
\hline Iniciação Científica & Orientações de Iniciação Científica \\
\hline Especialização & Orientações de Especialização \\
\hline Graduação & Orientações de Graduação \\
\hline
\end{tabular}

Fonte: Elaborado pelos autores.

A Tabela 5 evidencia os resultados das regressões dos determinantes das Pontuações dos docentes dos Programas de Pós-Graduação stricto sensu em Ciências Contábeis. 
Tabela 5 - Regressões

\begin{tabular}{|c|c|c|c|c|c|c|c|c|c|c|}
\hline & (1) & (2) & (3) & (4) & (5) & (6) & (7) & (8) & (9) & (10) \\
\hline \multirow[t]{2}{*}{ Idade } & -0.00 & -0.00 & -0.00 & -0.00 & -0.00 & -0.00 & -0.00 & -0.00 & -0.00 & 0.00 \\
\hline & $(0.01)$ & $(0.01)$ & $(0.01)$ & $(0.01)$ & $(0.01)$ & $(0.01)$ & $(0.01)$ & $(0.01)$ & $(0.01)$ & $(0.01)$ \\
\hline \multirow[t]{2}{*}{ Gênero } & & 0.11 & 0.12 & 0.13 & 0.13 & 0.11 & 0.11 & 0.11 & 0.11 & 0.18 \\
\hline & & (0.15) & $(0.15)$ & $(0.16)$ & (0.16) & (0.16) & $(0.16)$ & $(0.16)$ & $(0.16)$ & (0.16) \\
\hline \multirow[t]{2}{*}{ Mestrado } & & & $0.07^{* * * *}$ & $0.07^{* * * *}$ & $0.07^{* * *}$ & $0.07^{* * * *}$ & $0.07^{* * * *}$ & $0.07^{* * * *}$ & $0.07^{* * * *}$ & $0.09^{* * * *}$ \\
\hline & & & $(0.02)$ & $(\mathbf{0 . 0 3})$ & $(\mathbf{0 . 0 3})$ & $(0.03)$ & $(0.03)$ & $(\mathbf{0 . 0 3})$ & $(0.03)$ & $(0.02)$ \\
\hline \multirow[t]{2}{*}{ Doutorado } & & & & 0.04 & 0.06 & 0.06 & 0.07 & 0.06 & 0.06 & 0.01 \\
\hline & & & & $(0.09)$ & $(0.09)$ & $(0.09)$ & $(0.09)$ & $(0.09)$ & $(0.09)$ & $(0.09)$ \\
\hline \multirow[t]{2}{*}{ Pós-doutorado } & & & & & -0.12 & -0.12 & -0.11 & -0.09 & -0.09 & -0.05 \\
\hline & & & & & $(0.23)$ & $(0.23)$ & $(0.23)$ & $(0.23)$ & $(0.23)$ & (0.19) \\
\hline \multirow[t]{2}{*}{ Inic.Científica } & & & & & & $0.05^{*}$ & $0.06^{* *}$ & $0.05^{*}$ & $0.05^{*}$ & 0.03 \\
\hline & & & & & & $(0.03)$ & $(0.03)$ & $(\mathbf{0 . 0 3})$ & $(0.03)$ & $(0.03)$ \\
\hline \multirow[t]{2}{*}{ Especialização } & & & & & & & $0.02^{*}$ & 0.01 & 0.01 & 0.01 \\
\hline & & & & & & & $(0.01)$ & $(0.01)$ & $(0.01)$ & $(0.01)$ \\
\hline \multirow[t]{2}{*}{ Graduação } & & & & & & & & $\mathbf{0 . 0 3}^{* * * *}$ & $0.03^{* * * *}$ & 0.01 \\
\hline & & & & & & & & $(0.01)$ & $(0.01)$ & $(0.01)$ \\
\hline \multirow[t]{2}{*}{ _cons } & -0.06 & -0.14 & -0.14 & -0.14 & -0.14 & -0.15 & -0.19 & -0.25 & -0.25 & 0.46 \\
\hline & $(0.27)$ & $(0.29)$ & $(0.29)$ & $(0.29)$ & $(0.29)$ & $(0.29)$ & $(0.29)$ & $(0.29)$ & $(0.29)$ & $(0.39)$ \\
\hline $\begin{array}{c}N \\
\operatorname{adj} . R^{2}\end{array}$ & 530 & 530 & 530 & 530 & 530 & 530 & 530 & 530 & 530 & 530 \\
\hline
\end{tabular}

Fonte: Elaborado a partir dos dados da pesquisa.

Notas: Erros Padrões Robustos entre Parênteses * significativo a 10\%** significativo a 5\% e *** significativo a $1 \%$. Foram utilizados apenas efeitos fixos de aluno nessas regressões.

Os resultados apresentados na tabela 5 dão indícios de que a produção científica dos docentes dos Programas de Pós-Graduação stricto sensu, aumenta em média aproximadamente 7\% para cada orientação de mestrado. Esse resultado foi significativo em todos os modelos estimados. Cabe salientar, que dentre os programas avaliados a grande maioria deles possui apenas cursos de mestrado, o que poderia explicar a relevância desse fator.

Nessa mesma linha, verifica-se que as orientações de iniciação científica apresentam um efeito positivo na pontuação dos professores. Em média o fato de o docente ter orientado um aluno nessa modalidade acresce em $5 \%$ o número de pontos, o que pode ser observado nos modelos de (6) a (9).

Quanto às orientações de graduação, pode-se observar que o fato do docente orientar um aluno de graduação aumenta em média 3\% a pontuação. Esse resultado apresentou significância nos modelos de (8) e (9). Cabe mencionar, conforme tabela descritiva $\mathrm{n}^{\circ} 4$, que as orientações de graduação representaram $40,88 \%$ das orientações no período, no entanto, talvez este fato não tenha representado um aumento significativo na pontuação do docente, já que os cursos de graduação não têm como principal objetivo a produção científica. 
Referente às orientações de especialização, verifica-se que o fato do docente orientar um aluno dessa modalidade acresce em $2 \%$ a pontuação deste professor, o que pode ser observado no modelo (7).

Por outro lado, em relação às orientações de doutorado, não há indícios que o acréscimo de uma nova orientação, aumente a produção científica do docente. Esse fato, talvez possa estar relacionado a existência de apenas quatro programas de Pós-Graduação stricto sensu com o nível de Doutorado e a essa implementação tenha sido recente. Conforme tabela descritiva $\mathrm{n}^{\circ}$ 4, as orientações de Doutorado representaram apenas 3,65\% das orientações no período. O primeiro Programa com nível de Doutorado no Sul foi da FURB, implementado em 01/01/2008, seguido da UNISINOS, desde 01/01/2013, da UFSC, desde 01/01/2013 e por último a UFPR, desde 01/01/2014.

As estimativas coadunam com os estudos de Martins e Lucena (2014), quando questionados qual seria o principal meio para alcance da produção científica exigida pela CAPES, 27,4\% dos docentes responderam que seriam as orientações de dissertações e teses, o que representou o segundo motivo mais apontado, ficando atrás apenas dos grupos de pesquisa. Este fato, talvez demostre que as orientações de alunos colaborem para a concretização da produção científica do docente, uma vez que o aluno de mestrado/doutorado recebe um treinamento diferenciado o que credencia a realizar pesquisas dentro da linha de trabalho do professor orientador.

Em relação à idade, também não há indícios que a variação da idade influencie na produção científica, em estudo realizado por Rorstad e Aksnes (2015), os resultados também não confirmaram que a idade influencia na produtividade do pesquisador, já que em alguns grupos analisados a produção aumentou e em outros grupos a produção diminuiu conforme o pesquisador envelheceu.

Assim como a idade, em relação ao sexo, não há indícios de que as diferenças de gênero influenciem na produção científica. Contudo, em estudo realizado por Rorstad e Aksnes (2015), os autores concluíram que em média o gênero feminino produz $20 \%$ a menos que o sexo masculino, embora tenha sido encontrado variações significativas em relação a posição acadêmica.

Condizente com uma análise global dos achados evidenciados, o estudo corrobora com a literatura anterior, no sentido de apresentar características acadêmicas dos docentes que corroboram com a produção científica qualificada (BARBOSA; BARROS, 2010; IMASATO; PERLIN; BORENSTEIN, 2017; MARTINS; LUCENA, 2014; RORSTAD; AKSNES, 2015; SANTANA, NGANGA; LEAL, 2014). No entanto, no âmbito de docentes de Programas de 
Pós-Graduação Stricto Sensu em Contabilidade da região Sul do Brasil, fora comprovado que apenas as orientações de mestrado, especialização, iniciação científica e graduação influenciam significativamente um acréscimo na produção científica qualificada.

\section{Considerações finais}

Esta pesquisa teve por objetivo analisar se as características acadêmicas de docentes dos Programas de Pós-Graduação stricto sensu de Ciências Contábeis da região Sul influenciam na produção científica qualificada de artigos em periódicos. Para concretizar esse objetivo, coletaram-se as informações por meio dos Currículos Lattes e da Plataforma Sucupira dos 108 docentes dos Programas analisados. Primeiramente, realizou-se análise descritiva e posteriormente foi realizada a análise quantitativa, com a utilização da regressão com dados em painel.

A partir da análise descritiva, algumas das características dos docentes foram analisadas, em relação ao sexo, observa-se que permanece a predominância de docentes do sexo masculino nos Programas de Pós-Graduação stricto sensu em Ciências Contábeis. Na pesquisa de Santana, Nganga e Leal (2014) os resultados apontaram também a predominância de docentes do sexo masculino.

Consoante à qualificação dos docentes no nível de doutorado, observa-se que existe predomínio da formação na área de negócios, ou seja, os docentes possuem formação em: Ciências Contábeis, Administração, Finanças, Economia e Agronegócios e a minoria dos docentes possuem o Doutorado nas áreas de Educação, Engenharias e Psicologia.

Em relação às publicações científicas, percebe-se que os docentes dos Programas de Pós-Graduação stricto sensu da UFSC e da FURB foram mais produtivos no período analisado, representando juntos 55,20\% da pontuação total. Este fato, pode estar relacionando ao tempo de existência dos Programas, ao número de docentes vinculados aos Programas, bem como ao fato de possuírem os dois níveis, o Mestrado e o Doutorado, assim possuindo um quantitativo representativo de orientações nestes dois níveis, conforme a tabela descritiva $n^{\circ} 4$. Tais achados foram convergentes com a pesquisa de Santana, Nganga e Leal (2014) que também encontraram a centralização da produção dos docentes vinculados aos Programas da UFSC e FURB.

Em relação às orientações, observou-se que os docentes além de orientarem nos Programas de Pós-Graduação, possuem orientações na graduação e na iniciação científica. Torna-se pertinente mencionar que as orientações no nível de Doutorado não foram representativas em relação ao número total de orientações e este fato pode estar relacionado ao 
número reduzido de Programas com nível de Doutorado, bem como a implementação deste nível ter sido tardia, ocorrendo apenas a partir de 2008 e posteriormente em 2013, 2014 e 2015.

Quanto à análise quantitativa, após a utilização da regressão com dados em painel observou-se que as orientações de mestrado, seguidas das orientações de iniciação científica, graduação e especialização deram indícios de aumento da pontuação dos docentes dos Programas de Pós-Graduação stricto sensu. Em relação as orientações de mestrado, que representaram em média $7 \%$ de acréscimo na pontuação dos docentes, este fato pode estar associado ao quantitativo de Programas que possuem apenas o nível de mestrado, como os Programas da UNISINOS, UFPR, FURB e UFSC, e também a tardia implementação dos cursos de Doutorado que ocorreram após 2008. Ainda quanto a análise quantitativa, observou-se que a idade e o gênero não influenciaram na pontuação dos docentes.

No âmbito de implicações práticas, este estudo promove duas contribuições. A primeira, refere-se à exteriorização das características dos docentes vinculados aos Programas de PósGraduação stricto sensu. Estas informações podem ser úteis para os Programas realizarem o seu planejamento quanto às exigências da CAPES para a produção científica entre outros quesitos exigidos, além de compreender como os Programas estão qualificados e traçar objetivos de como melhorar.

A segunda contribuição sob a ótica de implicações práticas, consiste no próprio público estudado. Faz-se pertinente para os docentes compreender como as orientações impactam em suas pontuações, mediante as publicações científicas. Tal fato pode assumir um papel importante na remodelagem das orientações dos docentes, visando direcionar seus esforços prioritariamente para algum nível de escolaridade (doutorado, mestrado, especialização, iniciação científica ou grupos de pesquisa).

Na perspectiva de implicações teóricas, o estudo avança ao revisitar as discussões sobre produção científica dos docentes universitários, especificamente aos que compõe os Programas de Pós-Graduações Stricto Sensu em Contabilidade, da região Sul do Brasil. Os achados podem instigar a melhor compreender as relações das orientações dos docentes sobre suas pontuações decorrentes de publicações, bem como apontar caminhos para estudos que controlem as relações testadas com outras variáveis, como redes de cooperação e experiência internacional, por parte dos docentes.

As principais limitações da presente investigação consistem na delimitação geográfica, de forma a abarcar apenas uma das cinco regiões do Brasil. Em decorrência deste fator limitante, recomenda-se ampliar a amostra e realizar novas pesquisas com todos os docentes dos 
Programas de Pós-Graduação stricto sensu em Ciências Contábeis, uma vez que os Programas de Pós-Graduação stricto sensu em Ciências Contábeis estão em expansão.

\section{Referências}

BARBOSA, Glauber de Castro; BARROS, Fabrício de Oliveira. Perfil dos autores na produção científica em contabilidade: o caso do congresso USP de controladoria e contabilidade e do congresso ANPCONT. Enfoque: reflexão contábil, Paraná, v. 29, n. 3, p. 22-33, set./dez. 2010.

CAMERON, Adrian Colin; TRIVEDI, Pravin. Microeconometrics using stata. Texas: StataCorp, 2009.

COORDENAÇÃO DE APERFEIÇOAMENTO DE PESSOAL DE NÍVEL SUPERIOR (CAPES). Documento de área 2009. Fundação. Brasília, 2009. Disponível em: https://www.capes.gov.br/images/stories/download/avaliacao/ADMIN17jun10.pdf. Acesso em: 30 abr. 2019.

COORDENAÇÃO DE APERFEIÇOAMENTO DE PESSOAL DE NÍVEL SUPERIOR (CAPES). Documento de área 2016. Fundação. Brasília, 2016. Disponível em: http://www.capes.gov.br/images/documentos/Documentos_de_area_2017/27_ADMI_docarea 2016.pdf Acesso em: 30 abr. 2019.

COORDENAÇÃO DE APERFEIÇOAMENTO DE PESSOAL DE NÍVEL SUPERIOR (CAPES). Relatório da Avaliação Quadrienal 2017. Fundação. Brasília, 2017. Disponível em: http://capes.gov.br/images/stories/download/avaliacao/relatorios-finais-quadrienal2017/20122017-Administracao-quadrienal.pdf Acesso em: 14 jun. 2019.

COORDENAÇÃO DE APERFEIÇOAMENTO DE PESSOAL DE NÍVEL SUPERIOR (CAPES). Cursos Recomendados/Reconhecidos. Fundação. Brasília, 2018. Disponível em: https://www.capes.gov.br/pt/cursos-recomendados. Acesso em: 30 abr. 2019.

COTTINGHAM, Juliet; HUSSEY, Roger. Publishing in Professional Accounting Journals: Academic Institutional Performance 1987-96. British Accounting Review, Portsmouth, v. 32, n. 1, p. 101-114, 2000.

GARVEY, William D. Communication: the essence of science. London: Pergamon Press, 1979.

GIL, Antônio Carlos. Como elaborar projetos de pesquisa. 4. ed. São Paulo: Atlas, 2002.

IMASATO, Takeyoshi; PERLIN, Marcelo Scherer; BORENSTEIN, Denis. Análise do perfil dos acadêmicos e de suas publicações científicas em Administração. Revista de administração contemporânea, Rio de Janeiro, v. 21, n. 1, p. 62-83, jan./fev., 2017. Disponível em: https://www.scielo.br/pdf/rac/v21n1/1415-6555-rac-21-01-00062.pdf. Acesso em: 30 abr. 2019. 
LEITE FILHO, Geraldo Alemandro. Perfil da produção científica dos docentes e programas de Pós-Graduação em ciências contábeis no Brasil. Revista de Contabilidade e Controladoria, Curitiba, v. 2, n. 2, p. 1-13, maio/ago. 2010.

MARTINS, Gilberto de Andrade; THEÓPHILO, Carlos Renato. Metodologia da investigação científica para ciências sociais aplicadas. 3. ed. São Paulo: Atlas, 2018.

MARTINS, Orleans Silva; LUCENA, Wenner Lucena. Produtivismo acadêmico: As práticas dos docentes dos programas de pós-graduação em contabilidade. Advances in Scientific and Applied Accounting, São Paulo, v. 7, n.1, p. 66-96, 2014.

MESQUITA, Rafael Fernandes de et al. O triênio 2010-2012 e a nova avaliação de periódicos da CAPES. Revista de Educação, Ciência e Cultura, Canoas, v. 18, n. 2, p. 33-47, jul./dez. 2013.

MUELLER, Suzana Pinheiro Machado. A ciência, o sistema de comunicação científica e a literatura científica. In: CAMPELLO, B. S.; CENDÓN, B. V.; KREMER, J. M. Fontes de informação para pesquisadores e profissionais. Belo Horizonte: UFMG, 2000.

MURCIA, Flávia Cruz de Souza; ROSA, Carolina Aguiar; BORBA, José Alonso. Produção Científica em Ciências Contábeis: uma comparação entre a meta estabelecida pela CAPES e a publicação de artigos por parte dos docentes de Programas de Pós-Graduação. Revista Contabilidade, Gestão e Governança, Brasília, v. 16, n. 1, p. 68-81, jan./fev. 2013.

OLIVEIRA, Marcelle Colares. Análise dos Periódicos Brasileiros de Contabilidade. Revista Contabilidade \& Finanças, São Paulo, v. 13, n. 29, p. 68-86, 2002.

PELEIAS, Ivam Ricardo et al. Evolução do ensino da contabilidade no Brasil: uma análise histórica. Revista Contabilidade \& Finanças, São Paulo, v. 18, n. 1, p. 19-32, jun. 2007.

PELEIAS, Ivam Ricardo et al. Dez anos de pesquisa científica em controladoria no Brasil (1997-2006). Innovation \& Management Review, São Paulo, v. 7, n. 1, p. 193-217, jan./mar. 2010.

PERLIN, Marcelo Scherer. Getlattesdata: reading bibliometric data from lattes platform. 2019. Disponível em: https://CRAN.R-project.org/package=GetLattesData. Acesso em: 04 jun. 2019.

RORSTAD, Kristoffer; AKSNES, Dag W. Publication rate expressed by age, gender and academic position: a large-scale analysis of Norwegian academic staff. Journal of Informetrics, Amsterdã, v. 9, n. 2, p. 317-333, 2015.

SANTANA, Amanda Rosa de; NGANGA, Camila Soueneta Nascimento; LEAL, Edvalda Araújo. Perfil e produção científica dos docentes nos Cursos de Pós-Graduação Stricto Sensu em Ciências Contábeis. In: CONGRESSO UFSC DE CONTROLADORIA E FINANÇAS E INICIAÇÃO CIENTIFICA EM CONTABILIDADE, 5., São Paulo, 2014. Anais [...]. Florianópolis: UFSC, 2014.

SAVIANI, Dermeval. Pós-Graduação em educação no Brasil: trajetória, situação atual e perspectivas. Revista Diálogo Educacional, Curitiba, v. 1, n. 1, p. 1-95, jan./jun. 2000. 
SILVA, Harley Almeida Soares da et al. Programas de Pós-Graduação em contabilidade: análise da produção científica e redes de colaboração. Revista de Contabilidade e Organizações, Ribeirão Preto, v. 6, n. 14, p. 146-162, jan./abr. 2012.

SOARES, Sandro Vieira; RICHARTZ, Fernando; MURCIA, Fernando Dal-Ri. Ranking da Pós-Graduação em contabilidade no Brasil: análise dos programas de mestrado com base na produção científica em periódicos acadêmicos no triênio 2007-2009. Revista Universo Contábil, Blumenau, v. 9, n. 3, p. 55-74, jul./set. 2013.

SOARES, Sandro Vieira et al. Programas de Pós-Graduação em Contabilidade: semelhanças e diferenças da produção bibliográfica. Race: revista de administração, contabilidade e economia, Joaçaba, v.17, n. 2, p. 695-732, maio/ago. 2018. Disponível em:

https://portalperiodicos.unoesc.edu.br/race/article/view/16494. Acesso em: 04 jun. 2019.

SOUZA, Fábia Jaiany Viana; SILVA, Maurício Corrêa; ARAÚJO, Aneide Oliveira. Uma análise da produção científica da área de contabilidade governamental nos periódicos que utilizam o SEER e o SciELO. Revista de Contabilidade da UFBA, Salvador, v. 7, n. 2, p. 22-37, maio/ago. 2012.

VIEIRA, Amanda Martins; ENSSLIN, Sandra Rolim; SILVA, Harley Almeida Soares da. Perfil da produção científica dos docentes dos departamentos de contabilidade de três universidades federais do sul do Brasil. Enfoque: Reflexão Contábil, Maringá, v. 30, n. 3, p. 44-59, set./dez. 2011.

WAICZYK, Cleomir; ENSSLIN, Eduardo Rolim. Avaliação da produção científica de pesquisadores: mapeamento das publicações científicas. Revista Contemporânea de Contabilidade, Florianópolis, v. 10, n. 20, p. 97-112, 2013. Disponível em: https://periodicos.ufsc.br/index.php/contabilidade/article/view/2175-8069.2013v10n20p97. Acesso em: 04 jun. 2019.

YAMAMOTO, Oswaldo Hajime et al. Produção científica e "produtivismo": há alguma luz no final do túnel? Revista Brasileira de Pós-Graduação, Brasília, v. 9, n. 18, p. 727-750, dez. 2012. 